\section{(6) OPEN ACCESS} published online only. To view please visit the journal online (http://dx.doi.org/10.1136/ jclinpath-2013-201605).

${ }^{1}$ Medical Device Consultants of Ridgewood, LLC, Ridgewood, New Jersey, USA

${ }^{2}$ Department of Mathematics and Statistics, Washburn University, Topeka, Kansas, USA

\section{Correspondence to} Jeffrey Voigt, Medical Device Consultants of Ridgewood, LLC, 99 Glenwood Rd. Ridgewood, NJ 07450, USA meddevconsultant@aol.com

Received 6 March 2013 Accepted 6 May 2013 Published Online First 31 May 2013

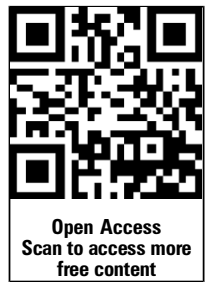

To cite: Voigt J, Mosier M. J Clin Pathol 2013:66:792796.

\title{
A powered bone marrow biopsy system versus manual methods: a systematic review and meta-analysis of randomised trials
}

\author{
Jeffrey Voigt, ${ }^{1}$ Michael Mosier ${ }^{2}$
}

\begin{abstract}
A significant number of bone marrow biopsies are performed annually. Outcomes of patient pain and sample size of biopsy are important issues as perception of patient pain is underestimated by clinicians and sample size assists in accurate diagnosis. Manual extraction of marrow biopsy is the main method used. Recently a powered system has been introduced. The objectives of this systematic review and meta-analysis were to determine if the powered system reduces patient pain and improves sample capture. A PubMed and Cochrane search for randomised controlled trials was undertaken comparing the powered system with manual methods. Risk of bias assessment and meta-analysis of outcomes using appropriate statistical methods were performed. Five randomised controlled trials were identified. Patient pain (measured via visual analogue scale (VAS) - 100 point scale) was significantly reduced using the powered system: mean difference $=-6.57$; $95 \% \mathrm{Cl}-12.93$ to $-0.22 ; p=0.04$. The relative reduction in pain was $17 \%-25 \%$ with the powered system. Sample biopsy size (length in $\mathrm{mm}$ ) was also significantly increased with the powered system: mean difference $=3.65 \mathrm{~mm} ; 95 \%$; $\mathrm{Cl} 1.61 \mathrm{~mm}$ to $5.68 \mathrm{~mm}$; $p=0.0005$. The relative increase in sample size was $33 \%$ with the powered system. Operator ease of use (as measured via VAS) and adverse events were similar. Despite limited operator experience, patients experienced less pain and sample sizes were increased without an increase in adverse events with the powered system. The powered system may offer an option in obtaining samples from patients whose pain is compromised by disease and may reduce redos.
\end{abstract}

\section{INTRODUCTION}

While bone marrow biopsy is a relatively common (600 000 US procedures) $)^{1-3}$ and straightforward procedure, there are issues associated with it, namely, patient morbidity (eg, haemorrhage, patient pain); adequacy of trephine length (and volume of biopsy sample); and ease of use for the clinician performing the procedure. It has been demonstrated in several trials that the greater the length (in millimetre) of the biopsy sample, the greater the likelihood of lesions being detected. ${ }^{45}$ It has also been cited in the literature that inadequate specimen size/amount requires a repeat procedure $^{6-9}$ Further, as it relates to the issue of patient pain, many clinicians do not recognise its severity and incidence. ${ }^{10}$ Last, the duration of a bone marrow biopsy procedure has been identified as the sole independent predictive factor for a patient's pain intensity. ${ }^{10}$
Bone marrow biopsy sampling technologies have until recently mainly consisted of a manual method. This method is most commonly carried out on the posterior superior iliac spine with a patient in the right or left lateral position. Local anaesthesia is provided via infiltrating an adequate area of the periosteum. Commonly a skin excision (with a lancet) is made prior to inserting the biopsy needle. With manual methods, once the needle contacts the bone, it is advanced by slowly rotating clockwise and counterclockwise until the cortical bone is penetrated and the marrow cavity is entered. Ideally the length of the biopsy specimen from an adult should measure at least $20 \mathrm{~mm}$ in length. ${ }^{8}$

Recently, a new battery-powered bone marrow biopsy system was developed (OnControl Vidacare Corporation, Shavano Park, Texas, USA) and cleared by the US Food and Drug Administration $(510[\mathrm{~K}]$ clearance number K072045). The OnControl system uses a battery-powered drill to insert the marrow needle into the iliac bone of adult haematology patients.

Several randomised controlled trials (RCTs) have been undertaken comparing the use of the powered bone marrow biopsy system with manual methods. However, the results from these studies have been equivocal. Further, the RCTs performed to date have been done on a relatively small number of patients. By combining like outcomes from multiple studies, a more definitive conclusion might be made on the utility of a powered bone marrow biopsy system.

The objective of this systematic review and meta-analysis is to determine whether the powered bone marrow biopsy system does provide for significantly different/improved outcomes on the endpoints of pain and sample size.

\section{MATERIALS AND METHODS}

RCTs comparing the powered system with manual biopsy methods were included. Participants included both patients with disorders of the blood and bone marrow. Patients were to never have had a previous bone marrow biopsy or other type of biopsies.

The outcome measures of importance that were evaluated were:

- Pain as measured via a visual analogue scale (VAS), either a 10 point or 100 point scale

- Bone marrow length (in $\mathrm{mm}$ ) or volume (in $\mathrm{mm}^{3}$ )

- Complications or adverse events (as defined by the trial)

- Length of time for the procedure (which as per above has been defined as an independent predictor of pain). ${ }^{10}$ 


\section{Search method}

The search method used can be found in online supplementary appendix 1.

\section{Data collection and analysis}

Data collection and analysis used a data collection form identified in online supplementary appendix 2. Data collection was first collected and analysed by one of the authors (JV). It was further adjudicated by the second author (MM) independently. Any disagreements were discussed between the two authors and a final agreement on including/excluding a trial was made by consensus.

\section{Assessment of risk of bias in included studies}

The risk of bias for each of the studies was reported and is summarised in the risk of bias figure and in online supplementary appendix 3 .

Two review authors independently assessed each included study for assessing risk of bias. ${ }^{11}$ A risk of bias table was completed for each eligible study. An assessment of risk of bias using a 'risk of bias summary figure' was compiled. This display of internal validity indicates the weight the reader may give the results of each study.

\section{Statistics}

Statistical analysis was performed using the statistical package that accompanies Cochrane Review Manager V.5.1. ${ }^{11}$ For continuous data (eg, pain scores, time of procedure, length of trephine), we used the mean difference (inverse variance, random or fixed effects model) if outcomes were measured in the same way between trials. The results of binary outcomes (ie, complication or not) were presented as risk ratios (Mantel-Haenszel, random or fixed effects model) with corresponding 95\% CIs.

Attempts were made to contact authors of included studies in order to obtain any missing data and/or to clarify biases. If data remained missing, we left it as missing and did not attempt to impute values. We assumed the data to be missing at random and therefore no bias would be introduced.

If trials could be combined, assessment of statistical heterogeneity was made using the $\mathrm{I}^{2}$ statistic in order to determine appropriateness for meta-analysis. If the $\mathrm{I}^{2}$ statistic was at or below $60 \%$, the heterogeneity was considered moderate and meta-analysis was deemed appropriate. If the value was greater than $60 \%$, sensitivity analyses were undertaken in an attempt to identify which studies were most likely causing the problem and are reviewed in the Discussion section. As well, in examining small size studies and heterogeneity, a comparison of fixed and random effects models was employed. If the estimates were similar, it would be concluded that any small-study effects would have little effect on the intervention effect estimate. ${ }^{11}$

Funnel plots were used to assess reporting bias for those studies combined for meta-analysis purposes. In the absence of bias, the funnel plot should approximate a symmetrical (inverted) funnel. ${ }^{11}$ If bias exists, an asymmetrical appearance of the funnel plot would exist. ${ }^{11}$ Each outcome was reported separately. Furthermore, an assessment was made of publication bias (including a review of unpublished studies), location bias (types of journals) and language bias.

\section{RESULTS}

\section{Results of the search}

The PRISMA flow diagram (figure 1) shows the results of the search.

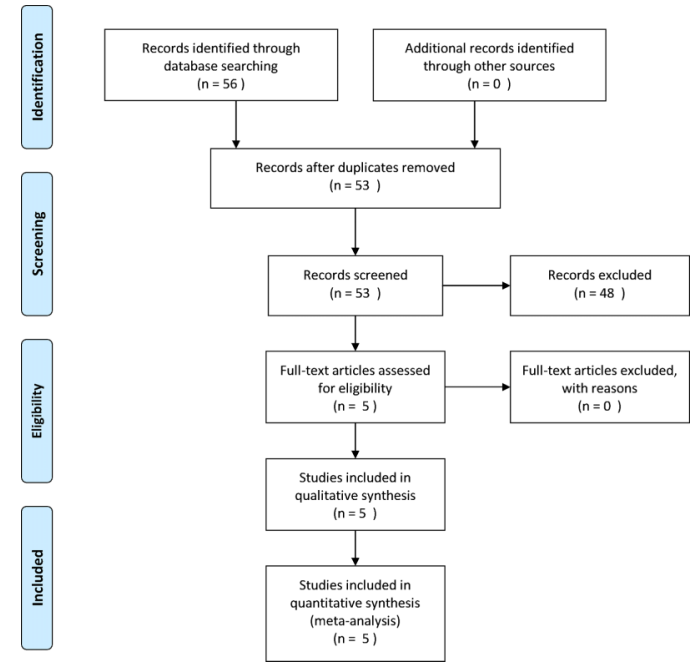

Figure 1 PRISMA flow diagram.

\section{Included studies}

Eight studies were included for further evaluation. ${ }^{12-19}$ Of these eight, two were excluded ${ }^{13} 18$ as they were abstracts of follow on published manuscripts. One additional study was excluded as it was an interim analysis of a single arm validation study. ${ }^{12}$

\section{Risk of bias in included studies}

The main risks of bias: blinding of operators was not possible between the two methods. Further, biases existed related to the experience levels of the operators between the two methodswith minimal to no experience with the powered system and significantly more expertise with the manual method (see figure 2 for risk of bias summary of trials; see online supplementary appendix 3 for additional details of each study). All funnel plots demonstrated symmetry.

\section{Effects of interventions}

In combining the five trials on the outcome of overall pain, as measured by the patient via the VAS, there was a significant difference in the pain score, favouring less pain with the powered bone marrow system: mean difference $=-6.57$; 95\% CI -12.93 to $-0.22 ; \mathrm{p}=0.04 ; \mathrm{I}^{2}=25 \%$ (figure 3 ) (inverse variance, random effects model). On a relative basis, this represents an approximate $17 \%$ reduction in overall pain over the average VAS score for manual biopsy of 39 . Further, three trials could be combined ${ }^{141619}$ on the outcome of pain upon needle insertion. A lessening of pain was observed with the powered bone marrow system: mean difference $=-7.87 ; 95 \%$ CI -15.77 to $0.44 ; \mathrm{p}=0.05 ; \mathrm{I}^{2}=23 \%$ (figure 4 ) (inverse variance, random effects model). On a relative basis, this represents an approximate $24 \%$ reduction in needle insertion pain over the average VAS score for manual biopsy of 33.5. Last, procedure time (in seconds) ${ }^{14} 1619$ was significantly less with the powered system:

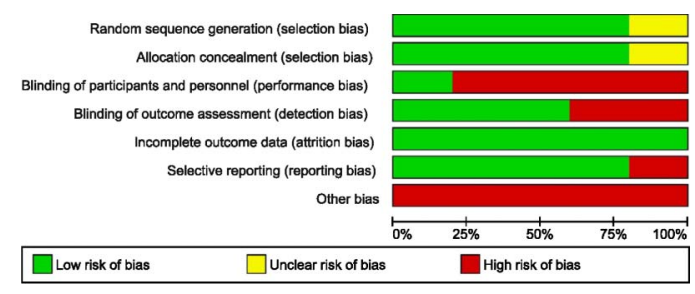

Figure 2 Risk of bias graph. 


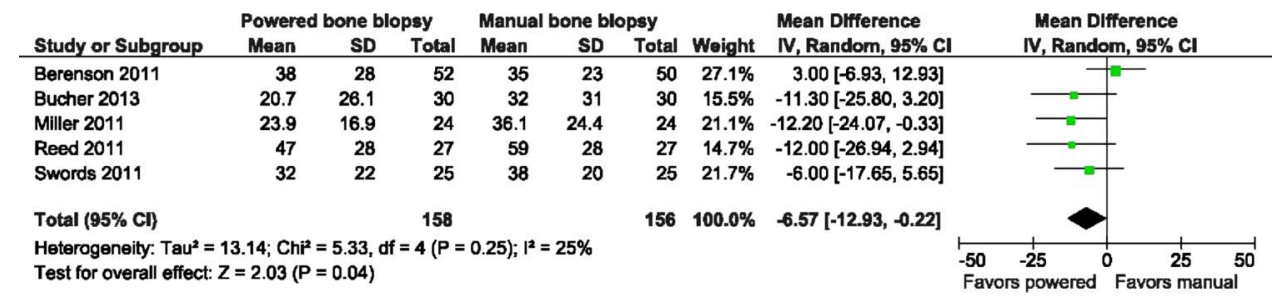

Figure 3 Forest plot visual analogue scale overall pain.

mean difference $=-85.35 \mathrm{~s} ; 95 \%$ CI $-145.14 \mathrm{~s}$ to $-25.56 \mathrm{~s}$; $\mathrm{p}=0.005 ; \mathrm{I}^{2}=89 \%$ (figure not shown) (inverse variance, random effects model).

Sample biopsy length as measured in millimetres $(\mathrm{mm})$ in all five studies was found to be significantly larger with the powered system: mean difference $=3.65 \mathrm{~mm}$; 95\%; CI $1.61 \mathrm{~mm}$ to $5.68 \mathrm{~mm} ; \mathrm{p}=0.0005$; although heterogeneity was moderately high at $\mathrm{I}^{2}=62 \%$ (figure 5) (inverse variance, random effects model). On a relative basis, this represents an approximate 33\% increase in the trephine length over the weighted average mean length for manual biopsy of $10.97 \mathrm{~mm}$. As well, in the two trials ${ }^{1419}$ that measured the overall volume in $\mathrm{mm}^{3}$, sample size was found to be significantly larger with the powered system: mean difference $=18.26 \mathrm{~mm}^{3} ; 95 \%$; CI $13.26 \mathrm{~mm}^{3}$ to $23.26 \mathrm{~mm}^{3} ; \mathrm{p}<0.00001 ; \mathrm{I}^{2}=0 \%$ (figure not shown) (inverse variance, random effects model).

As it relates to adverse events (as defined in the trial, ie, haematoma, extended pain, skin ensnared on shaft of needle), there was no statistically significant difference: risk ratio $=3.56$; $95 \%$ CI 0.59 to $21.42 ; \mathrm{p}=0.17 ; \mathrm{I}^{2}=0 \%$ (Mantel-Haenszel, random effects model) (figure not shown).

Last, as it relates to operator satisfaction, there was no statistically significant difference between the two methods in the two trials that measured this via a VAS: ${ }^{14}{ }^{19}$ mean difference $=1.41 ; 95 \%$; CI -0.75 to 3.56 ; $p=0.20$; although heterogeneity was high at $\mathrm{I}^{2}=95 \%$ (figure not shown).

\section{DISCUSSION}

Despite very limited experience (eg, $<5$ uses) by the majority of the operators using the powered bone marrow biopsy system involved in the randomised trials (compared with more extensive experience with manual biopsy methods), it was found that patients experienced significantly less overall pain and operators were able to obtain significantly larger sample sizes with the powered bone marrow biopsy system. Additionally, there was no difference in adverse outcomes between the two methods employed (powered vs manual). These results may speak of an ease of use issue measured in several of the trials (albeit differently) favouring the powered method.

The issue of pain reduction in patients undergoing bone marrow biopsy has been studied extensively in the literature and appears to be one of more important issues with bone marrow biopsy procedures, ${ }^{10}{ }^{20-22}$ which show that clinicians underestimate the level of pain patients experience. ${ }^{10}{ }^{22}$ A technology such as the powered system appears to assist in lowering the levels of pain experienced by patients and may help with this perception mismatch. When examined on a relative basis, this represents a $17 \%-24 \%$ reduction in pain when using the powered system, which could represent the difference between no pain and mild discomfort or mild discomfort and abject pain. This pain reduction may be especially helpful in patients whose pain threshold may be compromised due to disease states such as cancer and the associated treatments they undergo. Additionally, the significantly shorter time to perform the procedure, favouring the powered system, may aid in a reduction of pain.

The amount of bone marrow obtained is also important in helping pathologists accurately assess for the presence/absence of disease. It has been found in several studies that the larger the sample size, the greater the likelihood of accurately identifying lesions. ${ }^{45}$ Larger sample sizes have improved the 'positivity' of diagnosis by upwards of $13 \%-15 \% .^{5}{ }^{6}$ Further, the informative value of a bone marrow biopsy in detecting discrete marrow lesions is enhanced in a longer sample size due to the fact that it provides a large amount of marrow tissue for histological evaluation and more likely a sample from the innermost part of the bone marrow where abnormalities might be present. ${ }^{23}$ Again the powered system demonstrates that a significantly larger sample is obtained versus manual methods. As identified in the results section, the relative increase in sample trephine length of $33 \%$ with the powered system could be the difference in not having to repeat a biopsy and/or in accurately diagnosing a disease state. Last, the improved amount of sample capture with the powered system may reduce the need for repeat procedures as noted above.

Ease of use issues is also important for those performing the bone marrow biopsy. While not mentioned in the peer reviewed literature, performing manual biopsy procedures on a regular basis can result in operator problems such as carpal tunnel syndrome and wrist sprains, especially in patients with hard bones. ${ }^{24}$ Perhaps the powered system might alleviate this issue.

\section{Heterogeneity issues}

Two of the comparisons resulted in high heterogeneity between the studies: time in seconds for the procedure and sample length (in $\mathrm{mm}$ ). Regarding the time in seconds meta-analysis,

\begin{tabular}{|c|c|c|c|c|c|c|c|c|c|}
\hline \multirow[b]{2}{*}{ Study or Subgroup } & \multicolumn{3}{|c|}{ Powered bone blopsy } & \multicolumn{3}{|c|}{ Manual bone blopsy } & \multirow[b]{2}{*}{ Weight } & \multirow{2}{*}{$\begin{array}{l}\text { Mean Difference } \\
\text { IV, Random, } 95 \% \text { Cl }\end{array}$} & \multirow{2}{*}{$\begin{array}{l}\text { Mear } \\
\text { IV, Ra }\end{array}$} \\
\hline & Mean & SD & Total & Mean & SD & Total & & & \\
\hline Berenson 2011 & 31 & 31 & 52 & 32 & 29 & 50 & $35.1 \%$ & $-1.00[-12.65,10.65]$ & - \\
\hline Miller 2011 & 20.5 & 17.3 & 24 & 28.9 & 24.4 & 24 & $33.7 \%$ & $-8.40[-20.37,3.57]$ & \\
\hline Swords 2011 & 26 & 20 & 25 & 41 & 25 & 25 & $31.2 \%$ & $-15.00[-27.55,-2.45]$ & $=$ \\
\hline Total (95\% Cl) & & & 101 & & & 99 & $100.0 \%$ & $-7.87[-15.77,0.04]$ & 4 \\
\hline \multicolumn{8}{|c|}{$\begin{array}{l}\text { Heterogeneity: } \text { Tau }^{2}=11.03 ; \mathrm{Ch}^{2}=2.58, \mathrm{df}=2(P=0.27) ;\left.\right|^{2}=23 \% \\
\text { Test for overall effect: } Z=1.95(P=0.05)\end{array}$} & & $\begin{array}{l}-25 \\
s \text { power }\end{array}$ \\
\hline
\end{tabular}

Figure 4 Forest plot visual analogue scale pain needle insertion. 


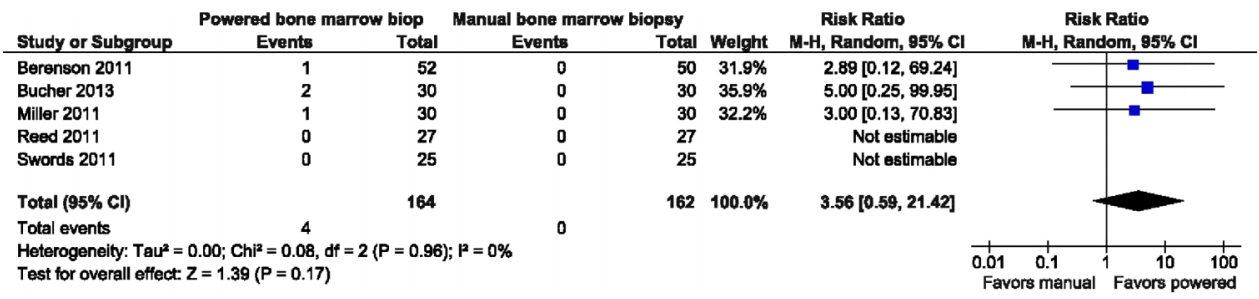

Figure 5 Forest plot overall trephine length $(\mathrm{mm})$.

the 2011 Miller $^{16}$ trial was excluded from the analysis with the heterogeneity statistic reduced to $0 \%$ (with findings significant at the $\mathrm{p}<0.00001$ level). Regarding the sample length size meta-analysis, the 2013 Bucher $^{15}$ study was excluded and the heterogeneity statistic reduced to $31 \%$ (with findings significant at the $\mathrm{p}<0.00001$ level). The findings from both Miller et al ${ }^{16}$ and Bucher et al ${ }^{15}$ while favouring the powered system were not as different in their magnitude as the other studies that were included. Further, additional analyses related to each, that is, pain (measured via a VAS) (with time in seconds of the procedure an independent predictor of pain) and volume of sample (measured in $\mathrm{mm}^{3}$, also related to sample length) demonstrated a significant difference, favouring the powered system. Based on these additional findings, it was assumed that the findings of increased sample length and time in seconds for the powered procedure were statistically different and the findings homogeneous.

\section{Quality of the evidence}

In evaluating the risk of bias graphs, the overall quality of the evidence might be considered average-with a similar amount of low and high risk of bias findings from all the studies. This review only included English language publications.

\section{CONCLUSIONS}

Despite limited operator experience, this systematic review and meta-analysis demonstrates a lowering of patient pain and an improved amount of sample capture with the powered system, with similar complications and adverse events. It also demonstrates, even with limited operator powered system experience, that it is easy to use.

\section{Take-home messages}

- There is less pain with the powered bone marrow biopsy system.

- There is greater bone marrow sample capture with the powered system for use in lesion evaluation.

- These findings were demonstrated despite limited experience with the powered system and.

- Studies with experienced powered bone marrow biopsy system users should be undertaken to confirm and reinforce these findings.

Contributors MM: conceived the review question, developed the protocol and performed part of writing or editing of the protocol and review. MM made an intellectual contribution to the review, and approved the final version and review prior to submission. JV: conceived the review question, developed the protocol and review, coordinated the protocol and review development, and completed the first draft of the protocol and review. JV made an intellectual contribution to the review, and approved the final version of the review prior to submission. MM: conceived the statistical analysis protocol and reviewed all of the statistical analysis prior to submission. Both authors are guarantors of the review.

Competing interests Jeff Voigt is a reimbursement consultant for the manufacturer of the powered bone marrow biopsy system. Michael Mosier has no interests to declare.

Provenance and peer review Not commissioned; externally peer reviewed.

Open Access This is an Open Access article distributed in accordance with the Creative Commons Attribution Non Commercial (CC BY-NC 3.0) license, which permits others to distribute, remix, adapt, build upon this work non-commercially, and license their derivative works on different terms, provided the original work is properly cited and the use is non-commercial. See: http://creativecommons.org/ licenses/by-nc/3.0/

\section{REFERENCES}

1 National Ambulatory Medical Care Survey. Division of healthcare statistics, national center for healthcare statistics (NCHS); Centers for Disease Control (CDC) 2010; http://www.cdc.gov/nchs/ahcd.htm (accessed 20 Jan 2013).

2 National hospital ambulatory medical care survey. Division of healthcare statistics, national center for statistics (NCHS); Centers for Disease Control (CDC) 2010; http:/l www.cdc.gov/nchs/ahcd.htm (accessed 20 Jan 2013).

3 Health care utilization project. HCUP Nationwide Inpatient Sample (NIS), Agency for Healthcare Research and Quality (AHRQ). 2010; http://hcupnet.ahrq.gov/ (accessed 20 Jan 2013).

4 Bain BJ. Bone marrow trephine biopsy. J Clin Pathol 2001;54:737-42.

5 Campbell JK, Matthews JP, Seymour JF, et al. Optimum trephine length in the assessment of bone marrow involvement in patients with diffuse large cell lymphoma. Ann Oncol 2003;14:273-6.

6 Bishop PW, McNally K, Harris M. Audit of bone marrow trephines. J Clin Pathol 1992;45:1105-8.

7 Reid MM, Roald B. Adequacy of bone marrow trephine biopsy specimens in children. J Clin Pathol 1996:49:226-9.

8 Lee $\mathrm{SH}$, Erber WN, Porwit A, et al. ISCH guidelines for the standardization of bone marrow specimens and reports. Int J Lab Hematol 2008;30:349-64.

9 Rimmer E. Adequacy of bone marrow trephine biopsy in a tertiary care center. Presentation at the Rocky Mountain American College Pathology meeting, November 14, 2008.

10 Kuball J, Schüz J, Gamm H, et al. Bone marrow punctures and pain. Acute Pain 2004;6:9-14.

11 Higgins JPT, Green S.eds Cochrane Handbook for Systematic Reviews and Interventions Version 5.1.0 [updated March 2011]. The Cochrane Collaboration 2011. http://www.cochrane-handbook.org

12 Berenson JR, Donahue DJ, Yellin 0, et al. A randomized trial evaluating a new powered bone marrow aspirate and core biopsy system compared to traditional manual devices. Blood 2009;114:Abstract 4234.

13 Berenson JR, Ori Y, Bojanower D, et al. A multicenter randomized clinical trial comparing a powered bone marrow biopsy system and manual bone marrow biopsy procedures. Blood 2010;116:Abstract 3840.

14 Berenson JR, Yellin O, Blumenstein B, et al. Using a powered bone marrow biopsy system results in shorter procedures, caused less residual pain to adult patients, and yields larger specimens. Diagn Pathol 2011;6. http://www.diagnosticpathology.org/ content/6/1/23

15 Bucher CM, Lehmann T, Tichelli A, et al. Comparison of a powered bone marrow biopsy device with a manual system: results of a prospective randomized trial. J Clin Pathol 2013:66:24-8.

16 Miller LJ, Philbeck TE, Montez DF, et al. Powered bone marrow biopsy procedures produce larger core specimens, with less pain, in less time than with standard manual devices. Hematol Rep 2011;3:22-5.

17 Reed LJ, Raghupathy R, Strakhan M, et al. The Oncontrol bone marrow biopsy technique is superior to the standard manual technique for hematologists-in-training: a prospective randomized trial. Hematol Rep 2011:3:60-4. 
18 Swords R, Anguita J, Higgins RA, et al. A new rotary powered device for bone marrow aspiration and biopsy yields superior specimens with less pain: results of a randomized clinical study. Blood 2010;116:Abstract 1529.

19 Swords RT, Anguita J, Higgins RA, et al. A prospective randomized study of a rotary powered device (OnControl) for bone marrow aspiration and biopsy. J Clin Pathol 2011;64:809-13.

20 Danhauer SC, Vishnevsky T, Campbell CR, et al. Music for patients with hematological malignancies undergoing bone marrow biopsy: a randomized controlled study of anxiety, perceived pain, and patient satisfaction. I Soc Integr Oncol 2010;8:140-7.
21 Hjortholm N, Jaddini E, Halaburda K, et al. Strategies of pain reduction during bone marrow biopsy. Ann Hematol 2013;92:145-9.

22 Lidén $\mathrm{Y}$, Olofsson $\mathrm{N}$, Landgren $\mathrm{O}$, et al. Pain and anxiety during bone marrow aspiration/biopsy: comparison of ratings among patients versus health-care professionals. Eur J Oncol Nurs 2012;16:323-9.

23 Islam A, Henderson ES. Value of a long-core biopsy in the detection of discrete bone marrow lesions. Histopathology 1988;12:641-8.

24 Symington $\mathrm{K}$, Martinez F. Bone marrow procedures move into the 21st century. Oncol News Int 2010;9. http://www.cancernetwork.com/print/article/10165/ 1654476? printable=true (accessed 27 Jan 2013) 


\section{Correction}

J Voigt, M Mosier. A powered bone marrow biopsy system versus manual methods: a systematic review and meta-analysis of randomised trials. J Clin Pathol 2013;66:9 792-796. Figure 5 in this paper was incorrect. It depicted adverse events identified and did not represent the overall trephine length analysis. The correct Figure 5 is shown below.

\begin{tabular}{|c|c|c|c|c|c|c|c|c|c|}
\hline \multirow[b]{2}{*}{ Study or Subgroup } & \multicolumn{3}{|c|}{ Powered bone biopsy } & \multicolumn{3}{|c|}{ Manual bone biopsy } & \multirow[b]{2}{*}{ Weight } & \multirow{2}{*}{$\begin{array}{l}\text { Mean Difference } \\
\text { IV, Random, } 95 \% \mathrm{Cl}\end{array}$} & \multirow{2}{*}{$\begin{array}{l}\text { Mean Difference } \\
\text { IV, Random, } 95 \% \mathrm{Cl}\end{array}$} \\
\hline & Mean & SD & Total & Mean & SD & Total & & & \\
\hline Berenson 2011 & 13.3 & 6.6 & 52 & 11 & 5.5 & 50 & $22.9 \%$ & $2.30[-0.05,4.65]$ & 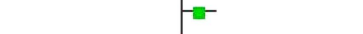 \\
\hline Bucher 2013 & 14.6 & 5.2 & 30 & 14.2 & 5.6 & 30 & $20.7 \%$ & $0.40[-2.33,3.13]$ & - \\
\hline Miller 2011 & 17 & 6.8 & 24 & 11.1 & 4.5 & 24 & $17.9 \%$ & $5.90[2.64,9.16]$ & -- \\
\hline Reed 2011 & 15.3 & 7.3 & 27 & 9.8 & 6.7 & 27 & $15.6 \%$ & $5.50[1.76,9.24]$ & 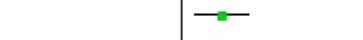 \\
\hline Swords 2011 & 13.1 & 4.8 & 25 & 8.2 & 3.6 & 25 & $22.9 \%$ & $4.90[2.55,7.25]$ & - \\
\hline Total $(95 \% \mathrm{Cl})$ & & & 158 & & & 156 & $100.0 \%$ & $3.65[1.61,5.68]$ & \\
\hline \multicolumn{9}{|c|}{$\begin{array}{l}\text { Heterogeneity: } \text { Tau }^{2}=3.28 ; \mathrm{Chi}^{2}=10.47, \mathrm{df}=4(P=0.03) ; I^{2}=62 \% \\
\text { Test for overall effect: } Z=3.51(P=0.0005)\end{array}$} & $\begin{array}{ccccc}-20 & -10 & 0 & 10 & 20 \\
\text { Favors manual } & \text { Favors powered }\end{array}$ \\
\hline
\end{tabular}

